\title{
Gaussian Measure of a Small Ball and Capacity in Wiener Space
}

Davar Khoshnevisan ${ }^{\mathrm{a} *}$ and Zhan $\mathrm{Shi}^{\mathrm{b}}$

${ }^{a}$ Department of Mathematics, University of Utah

Salt lake City, UT. 84112, USA.

Email: davar@math.utah.edu

${ }^{\mathrm{b}}$ L.S.T.A., Université Paris VI

4, Place Jussieu, 75252 Paris Cedex 05, France

Email: shi@ccr.jussieu.fr

Summary. We give asymptotic bounds for the Gaussian measure of a small ball in terms of the hitting probabilities of a suitably chosen infinite dimensional Brownian motion. Our estimates refine earlier works of Erickson [6].

Keywords. Small ball probability, capacity, Wiener space

1991 AMS Subject Classification. Primary. 60G15; Secondary. 60J45.

In honor of Professor M. Csörgő on occasion of his 65th birthday

\section{INTRODUCTION}

Let $(\mathcal{B},\|\cdot\|, \mu)$ denote a Gauss space in that $(\mathcal{B},\|\cdot\|)$ is a Banach space which carries a centered, Gaussian measure $\mu$ living on the Borel $\sigma$-field of $\mathcal{B}$.

An old problem, which has received much recent attention, is to describe the rate at which the $\mu$-measure of a small balanced ball (in $\mathcal{B}$ ) goes to zero, as the radius of the ball decreases to zero. To make this precise, suppose $p$ is a continuous semi-norm on $\mathcal{B}$. That is, $p: \mathcal{B} \mapsto \mathbb{R}$ such that

(i) For all $x, y \in \mathcal{B}, p(x+y) \leqslant p(x)+p(y)$;

(ii) for all $\alpha \in \mathbb{R}$ and all $x \in \mathcal{B}, p(\alpha x)=|\alpha| p(x)$;

(iii) whenever $x \rightarrow y$ in $\mathcal{B}, p(x-y) \rightarrow 0$.

In particular, note that $x \mapsto p(x)$ is a (nonnegative) continuous map from $\mathcal{B}$ into $\mathbb{R}_{+}$.

The so-called small ball problem for $\mu$ consists of finding a good approximation to the following:

$$
\mu_{p}(r) \triangleq \mu(\omega \in \mathcal{B}: p(\omega) \leqslant r)
$$

* Research supported by grants from the National Science Foundation and the National Security Agency 
as $r \rightarrow 0^{+}$. A major breakthrough is the recent work of Kuelbs and Li [11] relating $\mu_{p}$ to a combinatorial problem on the reproducing kernel Hilbert space corresponding to $\mu$. The latter route typically leads one to long-standing open problems in functional analysis; cf. [11] for details.

Throughout this paper, we only deal with the case when $p$ is transient. Rather than developing a theory for this, we define transience via the following technical assumption which will prevail throughout the rest of the paper:

Assumption 1.1. We assume the following two conditions:

(a) $p$ is transient in the sense that for some $\kappa>2$,

$$
\limsup _{r \rightarrow 0^{+}} r^{-\kappa} \mu_{p}(r)<\infty ; \text { and }
$$

(b) $p$ is nondegenerate in the sense that $1>\mu_{p}(1)>0$.

\section{Remark 1.1.1.}

(a) $\operatorname{Suppose} \operatorname{dim}(\mathcal{B})=d<\infty$. In other words, $\mathcal{B}$ is finite dimensional with (topological) dimension $d$. It is easy to see that as $r \rightarrow 0^{+}, \mu_{p}(r) \sim C r^{d}$ for some $C>0$. Therefore, Assumption 1.1(a) says that $d>2$ in this case. This corresponds to the well-known condition of transience in the classical sense.

(b) When $p$ has rank $\geqslant 3, p$ is transient; see [6] for details.

(c) If $p$ is nondegenerate, there exists $c>0$, such that $1>\mu_{p}(c)>0$. By considering the semi-norm $c^{-1} p$ instead, we see that Assumption 1.1(b) is not an essential restriction.

Motivated by the work of Erickson [6], this paper proposes a different approximation of $\mu_{p}$. To begin, recall that the triple $(\mathcal{B},\|\cdot\|, \mu)$ corresponds to a Wiener space $C$. To define it, let $C(\mathcal{B})$ denote the space of all continuous functions $\omega:[0,1] \mapsto \mathcal{B}$ with $\omega(0)=0$, endowed with the compact open topology. Let $\mathcal{C}$ denote the associated Borel field. For all $\omega \in C(\mathcal{B})$, let $B_{t}(\omega)=\omega(t)$. It is a well known fact that there exists a probability measure $\mathbb{P}$ on the measure space $(C(\mathcal{B}), \mathcal{C})$ which renders the process $B$ a $\mu$-Brownian motion; cf. Gross [8] or Üstünel [13] for a modern treatment as well as some of the new developments in this area. In particular, we mention the following important properties:

(i) with $\mathbb{P}$-probability one, $t \mapsto B_{t}$ is continuous;

(ii) $B$ has independent and stationary increments (under $\mathbb{P}$ );

(iii) for all $x \in \mathcal{B}^{*}$, the random variable $\left\langle x, B_{t}\right\rangle$ has a one-dimensional Gaussian distribution with mean 0 and variance $t \int_{\mathcal{B}}\|x\|^{2} \mu(d x)$ (under $\mathbb{P}$ );

(iv) $B$ is a $\mathcal{B}$-valued diffusion.

(v) $B_{0}=0, \mathbb{P}$-almost surely.

We will denote by $\mathbb{E}$ the expectation operator corresponding to the underlying (Gaussian) probability measure $\mathbb{P}$. 
ASYMP. METHODS IN PROB. STAT. (1998) B. Szyszkowicz (Editor) Elsevier Science B.V.

It will be convenient to write our results in terms of the $\mu$-Ornstein-Uhlenbeck process $O$ given by

$$
O_{t} \triangleq e^{-t / 2} B_{e^{t}}, \quad t \geqslant 0 .
$$

We note in passing the elementary fact that $O$ is a $\mathcal{B}$-valued stationary diffusion whose stationary measure is $\mu$.

For any $\varkappa>1$, define,

$$
\lambda_{p}(r ; \varkappa) \triangleq \sup \left\{a>0: \mu_{p}(a) \leqslant \varkappa \mu_{p}(r)\right\}, \quad r>0 .
$$

In the finite- dimensional case, it is possible to show that for any $\varkappa>0$, as $r \rightarrow 0^{+}$, $\lambda_{p}(r ; \varkappa) \sim \varkappa^{1 / d} r$, where $d \geqslant 3$ is the dimension of $\mathcal{B}$. In this connection, see also Remark 1.1.1(a).

The promised correspondence between $\mu_{p}$ and the process $O$ can then be described in terms of $\lambda_{p}$ as follows:

Theorem 1.2. Suppose $p: \mathcal{B} \mapsto \mathbb{R}$ is a nondegenerate, transient semi-norm on $\mathcal{B}$. Then, for all $T>0$, and all $\varkappa>1$, there exists a constant $c \in(1, \infty)$ such that for all $r \in(0,1 / c)$,

$$
\frac{\mu_{p}(r)}{c r^{2}} \leqslant \mathbb{P}\left(\inf _{0 \leqslant t \leqslant T} p\left(O_{t}\right) \leqslant r\right) \leqslant \frac{c \mu_{p}(r)}{\left(\lambda_{p}(r ; \varkappa)-r\right)^{2}} .
$$

In fact, the proof of Theorem 1.2 can be used, with little change, to show the following:

Corollary 1.3. Suppose $p: \mathcal{B} \rightarrow \mathbb{R}$ is a nondegenerate, transient semi-norm on $\mathcal{B}$. Then, for all $\lambda>0$ and all $\varkappa>1$, there exists a constant $c \in(1, \infty)$ such that for all $r \in(0,1 / c)$

$$
\frac{\mu_{p}(r)}{c r^{2}} \leqslant \int_{0}^{\infty} e^{-\lambda T} \mathbb{P}\left(\inf _{0 \leqslant t \leqslant T} p\left(O_{t}\right) \leqslant r\right) d T \leqslant \frac{c \mu_{p}(r)}{\left(\lambda_{p}(r ; \varkappa)-r\right)^{2}} .
$$

\section{Remark.}

(a) The quantity,

$$
\int_{0}^{\infty} e^{-\lambda T} \mathbb{P}\left(\inf _{0 \leqslant t \leqslant T} p\left(O_{t}\right) \leqslant r\right) d T
$$

is the $\lambda$-capacity of the "ball" $\{\omega \in \mathcal{B}: p(\omega) \leqslant r\}$; cf. Üstünel [13] and Fukushima et al. [7] for details. It turns out that under very general conditions, $\lambda_{p}(r ; \varkappa)-r$ has polynomial decay rate; cf. Remark 1.1.1(a) above. Thus, Theorem 1.2 and its variants provide exact (and essentially equivalent) asymptotics between the $\lambda$-capacity of a small ball and the small ball probability $\mu_{p}$ given by (1.1). 
ASYMP. METHODS IN PROB. STAT. (1998) B. Szyszkowicz (Editor) Elsevier Science B.V.

(b) In infinite dimensions - which is what is of interest here - the methods of Erickson [6] provide an upper bound of $\mu_{p}((1+\varepsilon) r)$ for any $\varepsilon>0$. In such cases, $\mu_{p}$ decays exponentially fast. Therefore, Theorem 1.2 is an essential improvement.

A refinement of Theorem 1.2 is proved in Section 2. Section 3 contains an explicit application. The methods of the latter section can be combined with those of [6] to provide a host of other examples.

\section{THE MAIN ESTIMATE}

In this section, we provide bounds for the probability that $O$ hits a small ball in terms of the small ball probability $\mu_{p}$ defined by (1.1). The main result of this section is the following probability estimate:

Theorem 2.1. Suppose $p$ is a continuous, nondegenerate, transient semi-norm on $\mathcal{B}$ in the sense of Assumption 1.1. Suppose further that $g: \mathbb{R}_{+} \mapsto \mathbb{R}_{+}$is a bounded measurable function satisfying: for some $\varkappa>1$,

$$
I_{g} \triangleq \liminf _{r \rightarrow 0^{+}} \frac{g(r)}{\left(\lambda_{p}(r ; \varkappa)-r\right)^{2}}>0 .
$$

Then, there exists a constant $c \in(1, \infty)$ which depends only on $\varkappa \sup _{x} g(x)$ and $I_{g}$, such that for all $r \in(0,1 / c)$,

$$
\frac{\mu_{p}(r) g(r)}{c r^{2}} \leqslant \mathbb{P}\left(\inf _{0 \leqslant t \leqslant g(r)} p\left(O_{t}\right) \leqslant r\right) \leqslant \frac{c \mu_{p}(r) g(r)}{\left(\lambda_{p}(r ; \varkappa)-r\right)^{2}} .
$$

\section{Remark 2.1.1.}

(a) The special case when $g(r) \equiv T$ immediately yields Theorem 1.2.

(b) Suppose $g \equiv T$ and $\operatorname{dim}(\mathcal{B})=d<\infty$. This is the finite dimensional version of Theorem 2.1 and is well known. Namely, that in $d>2$ dimensions, there exists a constant $c \in(1, \infty)$ such that for all $r$ small enough,

$$
c^{-1} r^{d-2} \leqslant \mathbb{P}\left(\inf _{0 \leqslant t \leqslant T} p\left(O_{t}\right) \leqslant r\right) \leqslant c r^{d-2} .
$$

(Recall Remark 1.1.1(a) regarding transience in finite dimensions as well as the estimates for $\lambda_{p}$.)

(c) The lower bound in Theorem 2.1 holds as long as $g$ is bounded and measurable. More precisely, the condition that $I_{g}>0$ is only needed for the upper bound.

(d) According to Weber [14] (cf. also Albin [1] and Khoshnevisan and Shi [9]), there is a connection between the modulus of continuity of a Gaussian process and its 
hitting probabilities. In our setting, the Gaussian process is infinite dimensional. In some infinite dimensional cases, such moduli of continuity are found; cf. Csáki and Csörgö [3] and Csáki et al. [4]. While it seems somewhat unlikely, one cannot help but ask if there is a connection between our results and such moduli of continuity in infinite dimensions.

The rest of this section is devoted to the proof of Theorem 2.1.

Lemma 2.2. Define,

$$
W_{t} \triangleq \frac{O_{t}-e^{-t / 2} O_{0}}{\sqrt{1-e^{-t}}} .
$$

For any fixed $t \geqslant 0, W_{t}$ as an element of $\mathcal{B}$ is an independent copy of $O_{0}$, satisfying,

$$
O_{t}=\sqrt{1-e^{-t}} W_{t}+e^{-t / 2} O_{0}
$$

Proof. By (1.2), $W_{t}=e^{-t / 2}\left(B_{e^{t}}-B_{1}\right)$ which is independent of $O_{0}=B_{1}$. To verify that $W_{t}$ is distributed as $O_{0}$, it suffices to show that for all $x \in \mathcal{B}^{*}$,

$$
\left\langle x, O_{t}\right\rangle-e^{-t / 2}\left\langle x, O_{0}\right\rangle \stackrel{(d)}{=} \sqrt{1-e^{-t}}\left\langle x, O_{0}\right\rangle
$$

This is a finite dimensional result which can be readily verified by checking means and covariances.

Lemma 2.3. Suppose $r>0$ and $t \geqslant 0$ are fixed and $p$ satisfies Assumption 1.1. Then uniformly over all $f \in \mathcal{B}$ with $p(f) \leqslant r$,

$$
\mathbb{P}\left(p\left(O_{t}\right) \leqslant r \mid O_{0}=f\right) \leqslant \mu_{p}\left(\sqrt{\frac{1+e^{-t / 2}}{1-e^{-t / 2}}} r\right)
$$

Proof. Let $W_{t}$ be as in (2.1). By properties of $p$,

$$
\sqrt{1-e^{-t}} p\left(W_{t}\right) \leqslant p\left(O_{t}\right)+e^{-t / 2} p\left(O_{0}\right)
$$

Therefore, conditional on $\left\{p\left(O_{0}\right) \leqslant r\right\},\left\{p\left(O_{t}\right) \leqslant r\right\}$ implies $\left\{p\left(W_{t}\right) \leqslant c r\right\}$, where

$$
c \triangleq \sqrt{\frac{1+e^{-t / 2}}{1-e^{-t / 2}}}
$$

Since by Lemma $2.2 p\left(W_{t}\right) \stackrel{(d)}{=} p\left(O_{0}\right)$, the result follows. 
Lemma 2.4. Suppose $p$ is a continuous semi-norm on $\mathcal{B}$ for which Assumption 1.1 is verified. Then for any $T>0$,

$$
\limsup _{r \rightarrow 0^{+}} r^{-2} \int_{0}^{T} \mu_{p}\left(\sqrt{\frac{1+e^{-t / 2}}{1-e^{-t / 2}}} r\right) d t<\infty .
$$

Proof. Note that for all $0<r<1$,

$$
\sqrt{\frac{1+e^{-t / 2}}{1-e^{-t / 2}}} r \leqslant 1, \quad \text { if and only if } \quad t \geqslant 2 \ln \left(\frac{1+r^{2}}{1-r^{2}}\right) .
$$

Therefore, for all $r>0$ small,

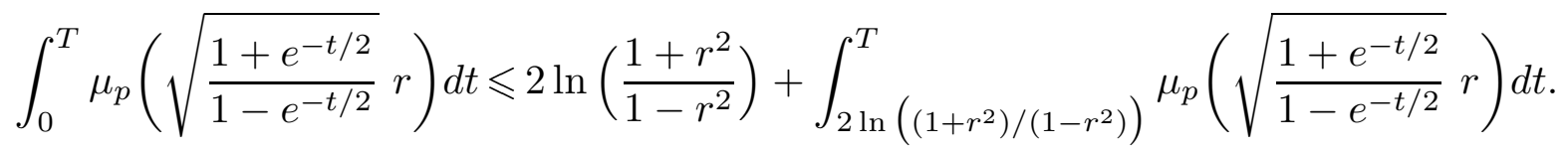

As $r \rightarrow 0^{+}$, the first term behaves like $4 r^{2}$. On the other hand, for $t \leqslant T$,

$$
\sqrt{\frac{1+e^{-t / 2}}{1-e^{-t / 2}}} \leqslant \frac{c_{1}}{\sqrt{t}}
$$

for some constant $c_{1}$. By Assumption 1.1(a), there exist $c_{2}, c_{3}, c_{4}>0$, such that for all $r>0$ small, the second term is bounded above by

$$
c_{2} \int_{c_{3} r^{2}}^{T}\left(\frac{r}{\sqrt{t}}\right)^{\kappa} d t \leqslant c_{4} r^{2}
$$

This concludes the proof.

Lemma 2.4 is sharp. Indeed, using Assumption 1.1(b) instead of 1.1(a) in the proof of Lemma 2.4, we immediately arrive at the following:

Lemma 2.5. Under the conditions of Lemma 2.4, for all $T>0$,

$$
\liminf _{r \rightarrow 0^{+}} r^{-2} \int_{0}^{T} \mu_{p}\left(\sqrt{\frac{1+e^{-t / 2}}{1-e^{-t / 2}}} r\right) d t>0 .
$$

We are ready to prove Theorem 2.1.

Proof of Theorem 2.1. Fix $\varkappa>1$ as given. The conditions on $g$ imply the existence of a constant $c \in(1, \infty)$, such that for all $r \in(0,1)$,

$$
c^{-1}\left(\lambda_{p}(r ; \varkappa)-r\right)^{2} \leqslant g(r) \leqslant c .
$$


ASYMP. METHODS IN PROB. STAT. (1998) B. Szyszkowicz (Editor) Elsevier Science B.V.

For all $r \geqslant 0$, define,

$$
\tau(r) \triangleq \inf \left\{s>0: p\left(O_{s}\right) \leqslant r\right\} .
$$

Since $p$ is continuous, $p^{-1}([0, r])$ is closed. Therefore, $\tau(r)$ is a stopping time for the diffusion $O$. By $(2.2)$ and the stationarity of $O$, for all $r \in(0,1)$,

$$
\begin{aligned}
g(r) \mu_{p}(r) & =\mathbb{E}\left[\int_{0}^{g(r)} \mathbb{1}\left\{p\left(O_{t}\right) \leqslant r\right\} d t\right] \\
& =\mathbb{E}\left[\int_{0}^{g(r)} \mathbb{1}\left\{p\left(O_{t}\right) \leqslant r\right\} d t \mathbb{1}\{0 \leqslant \tau(r)<g(r)\}\right] \\
& \leqslant \mathbb{E}\left[\int_{0}^{c} \mathbb{1}\left\{p\left(O_{t}\right) \leqslant r\right\} d t \mathbb{1}\{0 \leqslant \tau(r)<g(r)\}\right],
\end{aligned}
$$

where $\mathbb{1}\{\cdots\}$ is the indicator of whatever appears in the brackets. By Lemma 2.3,

$$
\begin{aligned}
\mathbb{P}\left(p\left(O_{t}\right) \leqslant r, \tau(r)=0\right) & =\mathbb{P}\left(p\left(O_{t}\right) \leqslant r \mid p\left(O_{0}\right) \leqslant r\right) \mathbb{P}(\tau(r)=0) \\
& \leqslant \mu_{p}\left(\sqrt{\frac{1+e^{-t / 2}}{1-e^{-t / 2}}} r\right) \mathbb{P}(\tau(r)=0) .
\end{aligned}
$$

Therefore,

$$
\mathbb{E}\left[\int_{0}^{c} \mathbb{1}\left\{p\left(O_{t}\right) \leqslant r\right\} d t \mathbb{1}\{\tau(r)=0\}\right] \leqslant \mathbb{P}(\tau(r)=0) \int_{0}^{c} \mu_{p}\left(\sqrt{\frac{1+e^{-t / 2}}{1-e^{-t / 2}}} r\right) d t .
$$

On the other hand, applying the strong Markov property at time $\tau(r)$, we arrive at the following:

$$
\begin{aligned}
& \mathbb{E}\left[\int_{0}^{c} \mathbb{1}\left\{p\left(O_{t}\right) \leqslant r\right\}\right. d t \\
&=\mathbb{1}\{0<\tau(r)<g(r)\}] \\
&=\mathbb{E}\left[\mathbb{1}\{0<\tau(r)<g(r)\} \int_{0}^{c-\tau(r)} \mathbb{1}\left\{p\left(O_{t}\right) \leqslant r\right\} d t \circ \theta(\tau(r))\right] \\
& \leqslant \mathbb{P}(0<\tau(r)<g(r)) \sup _{f} \mathbb{E}\left[\int_{0}^{c} \mathbb{1}\left\{p\left(O_{t}\right) \leqslant r\right\} d t \mid O_{0}=f\right],
\end{aligned}
$$

where $\theta$ is the shift functional on the paths of the diffusion $O$ and the supremum is over all $f \in \mathcal{B}$ with $p(f) \leqslant r$. By Lemma 2.3 ,

$$
\begin{aligned}
& \mathbb{E}\left[\int_{0}^{c} \mathbb{1}\left\{p\left(O_{t}\right) \leqslant r\right\}\right.d t \mathbb{1}\{0<\tau(r)<g(r)\}] \\
& \leqslant \mathbb{P}(0<\tau(r)<g(r)) \cdot \int_{0}^{c} \mu_{p}\left(\sqrt{\frac{1+e^{-t / 2}}{1-e^{-t / 2}}} r\right) d t .
\end{aligned}
$$


ASYMP. METHODS IN PROB. STAT. (1998) B. Szyszkowicz (Editor) Elsevier Science B.V.

Together with (2.5) and (2.4), this proves the following:

$$
g(r) \mu_{p}(r) \leqslant \mathbb{P}\left(\inf _{0 \leqslant t \leqslant g(r)} p\left(O_{t}\right) \leqslant r\right) \cdot \int_{0}^{c} \mu_{p}\left(\sqrt{\frac{1+e^{-t / 2}}{1-e^{-t / 2}}} r\right) d t .
$$

By Lemma 2.4, for all $r>0$ small, the right hand side is bounded above by a constant multiple of $r^{2} \mathbb{P}\left(\inf _{0 \leqslant t \leqslant g(r)} p\left(O_{t}\right) \leqslant r\right)$. In other words, we have proven the following:

$$
\liminf _{r \rightarrow 0^{+}} \frac{r^{2}}{g(r) \mu_{p}(r)} \mathbb{P}\left(\inf _{0 \leqslant t \leqslant g(r)} p\left(O_{t}\right) \leqslant r\right)>0 .
$$

This constitutes the first half of Theorem 2.1. We have also verified Remark 2.1.1(c). The second half proceeds along different lines.

Recall the definition of $\lambda_{p}$ from (1.3). Since $\mu_{p}$ is a decreasing function, one easily deduces that

(i) $r \mapsto \lambda_{p}(r ; \varkappa)$ is decreasing;

(ii) $\lambda_{p}(r ; \varkappa) \geqslant r$.

Fix $t \geqslant 0$. By Lemma 2.2 (in its notation), using the properties of $p$,

$$
p\left(O_{t}\right) \leqslant \sqrt{1-e^{-t}} p\left(W_{t}\right)+e^{-t / 2} p\left(O_{0}\right) .
$$

(This should be compared to the proof of Lemma 2.3). Observe that conditional on $\left\{p\left(O_{0}\right) \leqslant r\right\},\left\{p\left(W_{t}\right)<1\right\}$ implies the following:

$$
p\left(O_{t}\right) \leqslant \sqrt{1-e^{-t}}+r \leqslant t^{1 / 2}+r .
$$

Therefore, for all $t \leqslant\left(\lambda_{p}(r ; \varkappa)-r\right)^{2}$,

$$
\inf _{f} \mathbb{P}\left(p\left(O_{t}\right) \leqslant \lambda_{p}(r ; \varkappa) \mid O_{0}=f\right) \geqslant \mathbb{P}\left(p\left(W_{t}\right) \leqslant 1\right)=\mu_{p}(1)>0
$$

where the infimum is taken over all $f \in \mathcal{B}$ which satisfy $p(f) \leqslant r$. The rest of the proof follows from stationarity and the strong Markov property at time $\tau(r)$, viz.,

$$
\begin{aligned}
& 3 g(r) \mu_{p}\left(\lambda_{p}(r ; \varkappa)\right)=\mathbb{E}\left[\int_{0}^{3 g(r)} \mathbb{1}\left\{p\left(O_{t}\right) \leqslant \lambda_{p}(r ; \varkappa)\right\} d t\right] \\
& \geqslant \mathbb{E}\left[\int_{\tau(r)}^{3 g(r)} \mathbb{1}\left\{p\left(O_{t}\right) \leqslant \lambda_{p}(r ; \varkappa)\right\} d t \mid 0 \leqslant \tau(r) \leqslant g(r)\right] \cdot \mathbb{P}(0 \leqslant \tau(r) \leqslant g(r)) \\
& \geqslant \mathbb{E}\left[\int_{0}^{2 g(r)} \mathbb{1}\left\{p\left(O_{t}\right) \leqslant \lambda_{p}(r ; \varkappa)\right\} d t \circ \theta(\tau(r)) \mid 0 \leqslant \tau(r) \leqslant g(r)\right] \cdot \mathbb{P}(0 \leqslant \tau(r) \leqslant g(r)) \\
& \geqslant \inf _{f} \mathbb{E}\left[\int_{0}^{g(r)} \mathbb{1}\left\{p\left(O_{t}\right) \leqslant \lambda_{p}(r ; \varkappa)\right\} d t \mid O_{0}=f\right] \cdot \mathbb{P}(0 \leqslant \tau(r) \leqslant g(r)),
\end{aligned}
$$


where the infimum is taken over all $f \in \mathcal{B}$ such that $p(f) \leqslant r$ and $\theta$ is as before the shift on the paths of $O$. By (2.2),

$$
\begin{aligned}
& 3 g(r) \mu_{p}\left(\lambda_{p}(r ; \varkappa)\right) \\
& \quad \geqslant \inf _{f} \mathbb{E}\left[\int_{0}^{c^{-1}\left(\lambda_{p}(r ; \varkappa)-r\right)^{2}} \mathbb{1}\left\{p\left(O_{t}\right) \leqslant r\right\} d t \mid O_{0}=f\right] \mathbb{P}(0 \leqslant \tau(r) \leqslant g(r)),
\end{aligned}
$$

where the infimum is taken over all $f \in \mathcal{B}$ with $p(f) \leqslant r$. Since $c>1,(2.7)$ implies

$$
\begin{aligned}
3 g(r) \mu_{p}\left(\lambda_{p}(r ; \varkappa)\right) & \geqslant \mu_{p}(1) c^{-1}\left(\lambda_{p}(r ; \varkappa)-r\right)^{2} \mathbb{P}(0 \leqslant \tau(r) \leqslant g(r)) \\
& =\mu_{p}(1) c^{-1}\left(\lambda_{p}(r ; \varkappa)-r\right)^{2} \mathbb{P}\left(\inf _{0 \leqslant t \leqslant g(r)} p\left(O_{t}\right) \leqslant r\right) .
\end{aligned}
$$

By Assumption 1.1(b), $\mu_{p}(1)>0$. By (1.3), we have proven the following:

$$
\mathbb{P}\left(\inf _{0 \leqslant t \leqslant g(r)} p\left(O_{t}\right) \leqslant r\right) \leqslant \frac{3 c \varkappa}{\mu_{p}(1)} \frac{\mu_{p}(r) g(r)}{\left(\lambda_{p}(r ; \varkappa)-r\right)^{2}} .
$$

Together with (2.6), this proves Theorem 2.1.

\section{AN APPLICATION}

Recall the $\mu$-Brownian motion $B=\left(B_{t} ; t \geqslant 0\right)$ from Introduction. The goal of this section is to provide some estimates of the escape rates of $B$ when $p$ is transient. In light of Remark 1.1.1, it is not too difficult to convince oneself that under Assumption 1.1, $t \mapsto p\left(B_{t}\right)$ is transient in that $\mathbb{P}$-a.s., $\lim _{t \rightarrow \infty} p\left(B_{t}\right)=\infty$. It is the goal of this section to estimate the rate at which this blow-up occurs. We improve results of Erickson [6] concerning the following class of problems.

Assumption 3.1. We assume the existence of constants $r_{0}>0, K_{0}>1,0<\alpha \leqslant 2$, $\chi>0$ and $\beta \in \mathbb{R}$ such that for all $0<r \leqslant r_{0}$,

$$
K_{0}^{-1} r^{\beta} \exp \left(-\frac{\chi}{r^{\alpha}}\right) \leqslant \mu_{p}(r) \leqslant K_{0} r^{\beta} \exp \left(-\frac{\chi}{r^{\alpha}}\right)
$$

See Erickson [6], Li [12] and their combined references for many examples of when this assumption is valid.

Note that under Assumption 3.1, $\mathcal{B}$ is forced to be infinite-dimensional; see Remark 1.1.1. Moreover, Assumption 3.1 implies Assumption 1.1.

Our result on escape rates is the following: 
ASYMP. METHODS IN PROB. STAT. (1998) B. Szyszkowicz (Editor) Elsevier Science B.V.

Theorem 3.2. Suppose Assumption 3.1 is satisfied. Consider a measurable nonincreasing function $\psi: \mathbb{R}_{+} \mapsto \mathbb{R}_{+}$. Let

$$
\begin{aligned}
& I_{1}(\psi) \triangleq \int_{1}^{\infty} \psi^{\beta-\alpha}(t) \exp \left(-\frac{\chi}{\psi^{\alpha}(t)}\right) \frac{d t}{t}, \\
& I_{2}(\psi) \triangleq \int_{1}^{\infty} \psi^{\beta-2 \alpha-2}(t) \exp \left(-\frac{\chi}{\psi^{\alpha}(t)}\right) \frac{d t}{t} .
\end{aligned}
$$

Then

$$
\begin{aligned}
& I_{1}(\psi)=\infty \quad \Longrightarrow \mathbb{P}\left(p\left(B_{t}\right) \geqslant t^{1 / 2} \psi(t), \text { eventually for all } t \text { large }\right)=0 \\
& I_{2}(\psi)<\infty \Longrightarrow \mathbb{P}\left(p\left(B_{t}\right) \geqslant t^{1 / 2} \psi(t), \text { eventually for all } t \text { large }\right)=1
\end{aligned}
$$

Proof of (3.2). Assume $I_{1}(\psi)=\infty$. Define the Erdős sequence, $t_{k} \triangleq \exp (k / \log k)$. For simplicity, write $\psi_{k} \triangleq \psi\left(t_{k}\right)$. Furthermore, define the measurable event,

$$
E_{k} \triangleq\left\{p\left(B_{t_{k}}\right) \leqslant t_{k}^{1 / 2} \psi_{k}\right\}
$$

According to an argument of Erdös [5], there exists a constant $C>1$ such that,

$$
C^{-1}\left(\log \log t_{k}\right)^{-1 / \alpha} \leqslant \psi_{k} \leqslant C\left(\log \log t_{k}\right)^{-1 / \alpha} .
$$

By Brownian scaling,

$$
\mathbb{P}\left(E_{k}\right)=\mu_{p}\left(\psi_{k}\right) .
$$

Since $I_{1}(\psi)=\infty,(3.4)$ and Assumption 3.1 together yield:

$$
\sum_{k} \mathbb{P}\left(E_{k}\right)=\infty
$$

Suppose we could show

$$
\limsup _{N \rightarrow \infty} \frac{\sum_{k=1}^{N} \sum_{n=1}^{N-k} \mathbb{P}\left(E_{k} \cap E_{n+k}\right)}{\left(\sum_{k=1}^{N} \mathbb{P}\left(E_{k}\right)\right)^{2}}<\infty,
$$

then, according to Kochen and Stone $[10], \mathbb{P}\left(E_{k}\right.$, infinitely often $)>0$. The latter is a tail event. By the classical 0-1 law of Kolmogorov, $B_{t}$ has a trivial $\sigma$-field. Therefore, (3.5) implies $\mathbb{P}\left(E_{k}\right.$, i.o. $)=1$, which yields $(3.2)$.

It remains to prove (3.5). By Anderson's inequality (cf. [2]) and elementary Brownian properties (cf. Introduction),

$$
\begin{aligned}
\mathbb{P}\left(E_{k} \cap E_{n+k}\right) & =\mathbb{P}\left(E_{k} ; p\left(B_{t_{n+k}}-B_{t_{k}}+B_{t_{k}}\right) \leqslant t_{n+k}^{1 / 2} \psi_{n+k}\right) \\
& \leqslant \mathbb{P}\left(E_{k}\right) \mathbb{P}\left(p\left(B_{t_{n+k}}-B_{t_{k}}\right) \leqslant t_{n+k}^{1 / 2} \psi_{n+k}\right) \\
& =\mathbb{P}\left(E_{k}\right) \mu_{p}\left(\sqrt{\frac{t_{n+k}}{t_{n+k}-t_{k}}} \psi_{n+k}\right) .
\end{aligned}
$$


ASYMP. METHODS IN PROB. STAT. (1998) B. Szyszkowicz (Editor) Elsevier Science B.V.

From here, we can apply a classical argument going back at least to Erdös [5], and only provide its outline. Let $C_{i}(1 \leqslant i \leqslant 9)$ denote some unimportant constants. When $n \leqslant \log k, \sqrt{t_{n+k} /\left(t_{n+k}-t_{k}\right)} \psi_{n+k} \leqslant C_{1} n^{-1 / 2}$, which implies

$$
\mathbb{P}\left(E_{k} \cap E_{n+k}\right) \leqslant C_{2} \mathbb{P}\left(E_{k}\right) \exp \left(-C_{3} n^{\alpha / 2}\right) .
$$

For $\log k<n<(\log k)^{2}$, we have $t_{n+k} /\left(t_{n+k}-t_{k}\right) \leqslant C_{4}$, which yields

$$
\mathbb{P}\left(E_{k} \cap E_{n+k}\right) \leqslant C_{5} \mathbb{P}\left(E_{k}\right) \exp \left(-C_{6} \log k\right) \leqslant C_{5} \mathbb{P}\left(E_{k}\right) \exp \left(-C_{6} \sqrt{n}\right) .
$$

Finally, if $n \geqslant(\log k)^{2}$, then $t_{n+k} /\left(t_{n+k}-t_{k}\right) \leqslant 1+C_{7}(\log (n+k)) / n$. Hence

$$
\begin{aligned}
\mathbb{P}\left(E_{k} \cap E_{n+k}\right) & \leqslant C_{8} \mathbb{P}\left(E_{k}\right) \psi_{n+k}^{\beta} \exp \left(-\frac{\chi}{\psi_{n+k}^{\alpha}}\right) \\
& \leqslant C_{9} \mathbb{P}\left(E_{k}\right) \mathbb{P}\left(E_{n+k}\right) .
\end{aligned}
$$

Combining (3.6)-(3.8) gives (3.5).

To prove the other part of Theorem 3.2, we need a preliminary result.

Lemma 3.3. Under Assumption 3.1, there exist $\varkappa>1, r_{1}>0$ and $K_{1}>1$ such that for all $0<r \leqslant r_{1}$,

$$
r+K_{1}^{-1} r^{1+\alpha} \leqslant \lambda_{p}(r ; \varkappa) \leqslant r+K_{1} r^{1+\alpha}
$$

Proof. We will prove the upper bound for $\lambda_{p}(r ; \varkappa)$. The lower bound follows from similar arguments. Fix any $\gamma>0$. Two applications of (3.1) show that for all $0<r \leqslant r_{0}$ so small that $r+\gamma r^{1+\alpha} \leqslant r_{0}$,

$$
\begin{aligned}
\mu_{p}\left(r+\gamma r^{1+\alpha}\right) & \leqslant K_{0}\left(r+\gamma r^{1+\alpha}\right)^{\beta} \exp \left(-\frac{\chi}{\left(r+\gamma r^{1+\alpha}\right)^{\alpha}}\right) \\
& \leqslant K_{0}^{2}\left[\left(1+\gamma r_{0}^{\alpha}\right)^{\beta} \vee 1\right] \mu_{p}(r) \mathcal{Q}
\end{aligned}
$$

where,

$$
\mathcal{Q} \triangleq \exp \left(\frac{\chi}{r^{\alpha}}-\frac{\chi}{\left(r+\gamma r^{1+\alpha}\right)^{\alpha}}\right)
$$

A little calculus shows that whenever $\alpha>0$, for all $0<r \leqslant r_{0}$,

$$
1-\frac{1}{\left(1+\gamma r^{\alpha}\right)^{\alpha}} \leqslant\left(\left(1+\gamma r_{0}\right)^{\alpha-1} \vee 1\right) \alpha \gamma r^{\alpha}
$$

Hence,

$$
\mathcal{Q} \leqslant \exp \left(\chi \alpha \gamma\left(1 \vee\left(1+\gamma r_{0}\right)^{\alpha-1}\right)\right)
$$


ASYMP. METHODS IN PROB. STAT. (1998) B. Szyszkowicz (Editor) Elsevier Science B.V.

Fix any $\varkappa>K_{0}^{2}$. Note that for all $\gamma>0$ small,

$$
K_{0}^{2}\left[\left(1+\gamma r_{0}^{\alpha}\right)^{\beta} \vee 1\right] \exp \left(\chi \alpha \gamma\left[1 \vee\left(1+\gamma r_{0}\right)^{\alpha-1}\right]\right) \leqslant \varkappa
$$

Thus, we have shown that $\mu_{p}\left(r+\gamma r^{1+\alpha}\right) \leqslant \varkappa \mu_{p}(r)$. That is, for any $\varkappa>K_{0}^{2}$ and all $\gamma>0$ small enough, $\lambda_{p}(r ; \varkappa) \geqslant r+\gamma r^{1+\alpha}$.

Corollary 3.4. Suppose that Assumption 3.1 holds. For $K>0$, there exists a constant $c>1$ such that for all $r \in(0,1 / c)$,

$$
\begin{aligned}
c^{-1} r^{\beta+\alpha-2} \exp \left(-\frac{\chi}{r^{\alpha}}\right) & \leqslant \mathbb{P}\left(p\left(B_{t}\right) \leqslant t^{1 / 2} r, \text { for some } t \in\left[1,1+K r^{\alpha}\right]\right) \\
& \leqslant c r^{\beta-\alpha-2} \exp \left(-\frac{\chi}{r^{\alpha}}\right) .
\end{aligned}
$$

We are now ready to complete the proof of Theorem 3.2.

Proof of (3.3). Assume $I_{2}(\psi)<\infty$. Let $t_{k}$ and $\psi_{k}$ be as in the proof of (3.2). Define,

$$
\mathcal{U}_{k} \triangleq \mathbb{P}\left(p\left(B_{t}\right) \leqslant t_{k+1}^{1 / 2} \psi_{k}, \text { for some } t \in\left[t_{k}, t_{k+1}\right]\right)
$$

By Brownian scaling,

$$
\mathcal{U}_{k} \leqslant \mathbb{P}\left(p\left(B_{t}\right) \leqslant t^{1 / 2} \varphi_{k}, \text { for some } t \in\left[1, t_{k+1} / t_{k}\right]\right),
$$

where $\varphi_{k} \triangleq\left(t_{k+1} / t_{k}\right)^{1 / 2} \psi_{k}$. By $(3.4), t_{k+1} / t_{k}=1+O\left(\varphi_{k}^{\alpha}\right)$. Therefore, Corollary 3.4 shows that

$$
\mathcal{U}_{k} \leqslant C_{9} \varphi_{k}^{\beta-\alpha-2} \exp \left(-\frac{\chi}{\varphi_{k}^{\alpha}}\right) \leqslant C_{10} \psi_{k}^{\beta-\alpha-2} \exp \left(-\frac{\chi}{\psi_{k}^{\alpha}}\right)
$$

where $C_{9}$ and $C_{10}$ are two constants. Since $I_{2}(\psi)<\infty$, this yields $\sum_{k} \mathcal{U}_{k}<\infty$. By the Borel-Cantelli lemma, for all $k_{0}$ large enough (random but finite $\mathbb{P}$-a.s.), the following holds $\mathbb{P}$-a.s. for all $k \geqslant k_{0}$,

$$
\inf _{t_{k} \leqslant t \leqslant t_{k+1}} p\left(B_{t}\right) \geqslant t_{k+1}^{1 / 2} \psi_{k}
$$

Take any $k \geqslant k_{0}$ and $t \in\left[t_{k}, t_{k+1}\right]$. Then, by monotonicity,

$$
p\left(B_{t}\right) \geqslant t^{1 / 2} \psi(t)
$$

as desired. 
ASYMP. METHODS IN PROB. STAT. (1998) B. Szyszkowicz (Editor) Elsevier Science B.V.

\section{REFERENCES}

1. J.M.P. Albin (1992), On the general law of iterated logarithm with application to selfsimilar processes and to Gaussian processes in $\mathbb{R}^{n}$ and Hilbert space, Stoch. Proc. Appl. 41, 1-31.

2. T.W. Anderson (1955), The integral of symmetric unimodular functions over a symmetric convex set and some probability inequalities, Proc. Amer. Math. Soc. 6, 170-176.

3. E. Csáki and M. Csörgö (1992), Inequalities for increments of stochastic processes and moduli of continuity, Ann. Prob. 20, 1031-1052.

4. E. Csáki, M. Csörgő and Q.M. Shao (1992), Fernique type inequalities and moduli of continuity for $\ell^{2}$-valued Ornstein-Uhlenbeck processes, Ann. Inst. H. Poincaré Prob. Statist. 28, 479-517.

5. P. Erdős (1942), On the law of the iterated logarithm, Ann. Math. 43, 419-436.

6. K.B. Erickson (1980), Rates of escape of infinite dimensional Brownian motion, Ann. Prob. 8, 325-338.

7. M. Fukushima, Y. Oshima and M. Takeda (1994), Dirichlet Forms and Symmetric Markov Processes, Walter de Gruyter, New York.

8. L. Gross (1965), Abstract Wiener space, Proc. Fifth Berkeley Symp. Math. Statist. Prob. II, Part 1, Univ. California Press, Berkeley, 31-41.

9. D. Khoshnevisan and Z. Shi (1996), Hitting estimates for Gaussian random fields, (Unpublished report).

10. S.B. Kochen and C.J. Stone (1964), A note on the Borel-Cantelli lemma, Illinois J. Math. 8, 248-251.

11. J. Kuelbs and W.V. Li (1993), Metric entropy and the small ball problem for Gaussian measures, J. Funct. Anal. 116, 133-157.

12. W.V. Li (1992), Comparison results for the lower tail of Gaussian seminorms, J. Th. Prob. 5, 1-31.

13. A.S. Üstünel (1995), An Introduction to Analysis on Wiener Space, Springer, Berlin.

14. M. Weber (1983), Polar sets of some Gaussian processes, Probability in Banach Spaces IV, Lecture Notes in Mathematics 990, Springer, Berlin, 204-214. 